\title{
IMPACTO SOCIOAMBIENTAL DE LA INDUSTRIA PETROLERA en TABAsCo: el CASO DE LA ChONTALPA
}

\author{
Manuel Jesús Pinkus-Rendón \\ Alicia Contreras-Sánchez
}

\begin{abstract}
Resumen: Se emprendió esta investigación con el objetivo de valorar los impactos ambientales, económicos, sociales y políticos derivados de las actividades de Petróleos Mexicanos (PEMEX) en cuatro poblaciones del estado de Tabasco. Se enmarca el estudio en la dinámica social comunitaria derivada de las actividades de la petrolera con la finalidad de conocer cómo afectan a los habitantes las relaciones con PEMEX y con los gobiernos locales. Partimos de la hipótesis de que el deterioro ambiental, históricamente asociado con la transformación del tejido social, tiene que analizarse en un contexto social más amplio considerando las transformaciones que la política económica neoliberal está generando en el país, y en especial en el ámbito local, a partir de los años ochenta del siglo $X X$.
\end{abstract}

Palabras clave: Petróleo, impacto socioambiental, campesinos.

Enviado a dictamen: 29 de febrero de 2012 Aprobación: 25 de julio de 2012

Revisiones: 1

Dr. Manuel Jesús Pinkus Rendón, doctor en Estudios Mesoamericanos por la UNAM. Labora en la Universidad Autónoma de Yucatán, Centro de Investigaciones Regionales, Unidad de Ciencias Sociales. Temas de especialización: globalización, desarrollo, sustentabilidad, medioambiente y turismo. Correo electrónico: yucpinkus@yahoo.com.mx; mpinkus@audy.mx.

Dra. Alicia Contreras Sánchez, doctora en Historia por el COLMICH. Labora en la Universidad Autónoma de Yucatán, Centro de Investigaciones Regionales, Unidad de Ciencias Sociales. Temas de especialización: historia económica y social, historia demográfica de la colonia al porfiriato. Correo electrónico: csanchez@uady.mx.
Abstract: This research was undertaken in order to assess the environmental, economic, social and political challenges posed by the activities of Petróleos Mexicanos (PEMEX) in four towns in the state of Tabasco. Study is framed in community social dynamics derived from petroleum activities in order to know how they affect people with PEMEX and relations with local governments. We hypothesized that environmental degradation, historically associated with the transformation of the social fabric, must be analyzed in a broader social context considering the changes that neoliberal economic policy being generated in the country, especially at the local level, since the eighties of the $X X$ century.

Keywords:Petroleum, socio-envitommental impacts, peasant imaginaries.

\section{Introducción}

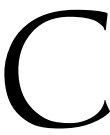

on el objeto de comprender la tendencia y el comportamiento de los conflictos generados por las actividades de Petróleos Mexicanos (PEMEX) en Tabasco, emprendimos una investigación cuyo objetivo general fue el estudio y la valoración de los impactos ambientales, económicos, sociales y políticos, así como en el tejido social de cuatro poblaciones de la entidad, en las cuales la actividad 
petrolera se ha ido expandiendo durante los últimos dos años, particularmente a través del nuevo proyecto de explotación de pozos petroleros en Cárdenas, Tabasco. El estudio de estos procesos lo enmarcamos dentro de la dinámica social comunitaria, con la finalidad de conocer cómo ésta ha sido afectada en sus relaciones con PEMEX y los gobiernos locales.

Partimos dela hipótesis de queel deterioro ambiental, asociado históricamente con la transformación del tejido social, tiene que ser analizado como parte de los procesos de un contexto estructural más amplio, particularmente con las transformaciones generadas en el país por la política económica neoliberal impulsada por el Gobierno mexicano desde la segunda mitad de los años ochenta del siglo XX y, en especial, en el ámbito local de las poblaciones que integran las diversas regiones del estado de Tabasco.

En este artículo identificamos los impactos y efectos que la expansión petrolera ha generado en la calidad de vida de cuatro comunidades rurales: José Ma. Pino Suárez C-22, Veinte de Noviembre C-33, Ing. Eduardo Chávez Ramírez C-27 y la Colonia Ingenio Benito Juárez, todas ellas del municipio de Cárdenas, Tabasco, con la intención de determinar de modo cualitativo el vínculo entre el deterioro ambiental y la actual situación socioeconómica de estas comunidades que se encuentran asentadas en las áreas petroleras a desarrollar por la paraestatal. Asimismo, damos cuenta sobre sus efectos en las actividades del sector primario de la economía regional, en el ecosistema y en otros ámbitos del entorno social.

Hoy en día, muchos problemas locales como el incremento de la pobreza, señalados por las autoridades públicas y muy manifiestos en el interior de las comunidades, tienen relación con las condiciones prevalecientes en el ambiente natural, marcado por un significativo deterioro y las restricciones en el acceso de los pobladores originarios a los recursos naturales. Otro objetivo fundamental de este trabajo socioambiental es presentar la "voz"1 de los trabajadores rurales con respecto a la pérdida de sus recursos naturales, de los cuales habían sido usufructuarios por varias generaciones.

El área de nuestro estudio se encuentra ubicada en el sureste de México, en los territorios que tradicionalmente eran reconocidos como asiento de los llamados pueblos mayas de muy diversos grupos lingüísticos, los cuales han variado de manera significativa debido a diversas condiciones, como las derivadas de la presión demográfica ocasionada por el proceso de colonización de las tierras agostadas impulsado por el gobierno estatal hacia la década de los años setenta del siglo XX; el deterioro generalizado que este proceso profundizó en el ámbito rural; y la globalización económica neoliberal que asumió el Gobierno mexicano. Cabe señalar que este macro proceso impuso a los gobiernos de los países en desarrollo el abandono del apoyo económico al campo, con su consecuente empobrecimiento como resultado de las reformas al artículo 27 constitucional y a la Ley Agraria. Todos ellos fueron acontecimientos estructurales que generaron cambios socioeconómicos en el sector rural tabasqueño y crearon una creciente migración de los pueblos mayas hacia el interior del estado, hacia otros estados del sur sureste del país y, principalmente, hacia Estados Unidos y Canadá (Pinkus, 2010: 30-31) (ver mapa 1).

\section{Breve antecedente del desarrollo económico de la región de La Chontalpa}

Con el objeto de incorporar la región de la Chontalpa tabasqueña a un proyecto de desarrollo agropecuario, en 1965, el Gobierno federal proveyó a la Comisión del Río Grijalva, dependencia de la Secretaría de Agricultura y Recursos Hidráulicos (SARH), de instrumentos legales y financieros para la ejecución de infraestructuras que creasen las condiciones necesarias para el desarrollo de los programas agropecuarios, por medio de los cuales 
se buscaba el mejoramiento de la economía estatal y de las poblaciones, tales como:

- Construcción de presas de control,

- canales de riego y drenaje,

- agua potable,

- alcantarillado,

- desecación de pantanos,

- campañas contra plagas,

- apertura de nuevas vías de comunicación y

- proyectos de fomento agrícola, entre otros.

Cabe aclarar que estas transformaciones no fueron exclusivas del Plan Chontalpa, sino también de los proyectos de la Comisión del Río Grijalva (Pinkus y Contreras, 2012: 51).

El propósito de este programa de desarrollo fue incorporar 352 mil hectáreas de terrenos a la producción de cultivos como arroz, cacao, maíz, plátano y cítricos. Estas tierras fueron divididas en secciones de cinco mil hectáreas cada una, para que fueran otorgadas en propiedad ejidal a grupos de 500 familias de campesinos. En su aplicación, este Plan Chontalpa pasó por diversas etapas y enfrentó distintas dificultades en su desarrollo que exigieron ajustes y modificaciones en los distintos momentos de su existencia (Ortiz, 1987: 118) (ver mapa 2).

Es importante mencionar que en sus inicios el Plan Chontalpa, así como los proyectos de la Comisión del Grijalva, tuvieron resultados importantes en cuanto al mejoramiento de las condiciones de vida de las poblaciones involucradas, principalmente los expresados en las obras de infraestructura que se crearon para propiciar circunstancias favorables al desarrollo que el Gobierno estaba impulsando, tales como: servicios de salud y educación, desmontes para las obras de infraestructura (drenes, caminos y carreteras), redistribución de la población campesina, obras de beneficio social, construcción de poblados y viviendas en núcleos organizados con todos los servicios, investigación agropecuaria y asistencia técnica. Sin embargo, la producción y productividad agropecuaria no corrieron con la misma suerte, toda vez que continuó su declive, mientras que el crecimiento poblacional aumentó de modo sostenido hasta la década de los sesenta, lo cual dio como resultado la necesidad de poner en operación el Plan Chontalpa. ${ }^{2}$

No obstante, se observó un aumento significativo del movimiento poblacional en Cárdenas y Comalcalco por ser estos municipios, además de Centla, Paraíso y Macuspana - por Ciudad PEMEX-, de los más proclives para la explotación petrolera. Esta actividad atrajo hacia estas localidades a la fuerza de trabajo desocupada de otros municipios de la entidad y de otros estados de la República, tal como aparece en la siguiente gráfica, en la que es posible observar que el mayor pico del movimiento poblacional ocurrió desde la década de los noventa y, sobre todo, desde el año 2000 (INEGI, 2010) (ver gráfica l).

\section{El auge petrolero}

La petrolización de Tabasco puso de manifiestoel fracaso de grandes planes de diversificación agropecuaria, amén de que influyó en la disminución de las tierras dedicadas a estas actividades dentro del sector primario de la economía. El impulso de la actividad petrolera inició en los años cincuenta y se expandió de forma explosiva durante los años setenta del siglo XX. ${ }^{3}$ Como hemos observado en el cuadro anterior, durante este período aconteció una intensa movilidad social interna y desde otros estados de la República Mexicana: población procedente de Michoacán, Veracruz y Chiapas llegó a Tabasco y con este proceso se gestó el predominio del trabajo industrial asalariado en la región de La Chontalpa.

La petrolización del estado cobró auge y se descubrieron varios campos de potencial explotación, tales como: Fortuna Nacional, en 1949; Tortuguero, en 1950; José Colomo, en 1951. El primero y último campo de explotación darían origen, en 1974, a la creación 
de Ciudad PEMEX, la cual generaría más de 30\% de la producción nacional de gas (Martínez, 1996).

También fueron surgiendo otros centros de explotación petrolera como: La Venta, Usumacinta, Almendro, Santuario, San Román, Tintal, Samaria y Tucán, entre otros. Los pozos en operación fueron en aumento y dieron lugar a un fenómeno: el boom petrolero, que rebasó el auge de la producción platanera de agroexportación y se convirtió en la causa de apogeo socioeconómico en la región. Como en otros casos, los ingresos derivados de la exportación petrolera fueron manejados por la administración pública federal de Petróleos Mexicanos (Martínez, 1996).

Con el descubrimiento de grandes yacimientos petrolíferos en las regiones de Chiapas y Tabasco en 1973, las reservas del país pasaron de 6,000 millones de barriles, en 1975, a 60 mil millones en 1980. En 1974, debido a las nuevas reservas, México pasó de ser importador de crudo en el primer semestre de este año, a exportador en el segundo semestre (Martínez, 1996). El auge del petróleo en el estado de Tabasco coadyuvó al crecimiento de la población, de tal forma que mientras en 1980 había 1,062,961 habitantes, para 1990 se incrementó a 1,501,183 y para 2005 la población alcanzó la cifra de 1,989,969, por lo que en 25 años el aumento poblacional fue de $25 \%$ (488,786 personas de 1990 a 2005) debido al crecimiento poblacional natural y a los movimientos migratorios.

El crecimiento anual de la población fue 4.2\%, mientras la producción de alimentos aumentó apenas 0.7\% debido a que, paradójicamente, la superficie cosechada disminuyó de 178,000 hectáreas a 164,000, porque la población joven trabajadora se volcó hacia la explotación petrolera, o bien, en muchos casos, migró hacia otros estados del sureste, lo que trajo como consecuencia la dependencia de la población local del mercado regional y nacional para el suministro de los productos básicos en su alimentación. Por su parte, la producción petrolera aumentó de 92,000 a casi 600,000 barriles diarios en tan solo 20 años (Martínez, 1996).
En la actualidad, la población del estado se concentra en ocho de los 17 municipios de la entidad tabasqueña, aquellos que están más dedicados a la explotación petrolera como Cárdenas, Centla, Paraíso, Comalcalco (el de mayor crecimiento), Macuspana y Ciudad PEMEX. De los 19,000 trabajadores contratados por PEMEX en 1980, sólo 41\% eran tabasqueños y el restante 59\% eran procedentes de otros estados, trasladados por la paraestatal a esta entidad. Entre este año y 1983 el número de trabajadores de la industria se incrementó en $83 \%$, con lo que poco más de 25,000 personas trabajaban para PEMEX. Sólo para dar una idea del personal empleado, puede decirse que durante año y medio, tiempo que duró la construcción del puerto de altura de Dos Bocas, la empresa dio empleo a 20,000 obreros.

\section{Los primeros cambios}

Los cambios operados en Tabasco fueron drásticos porque la inversión federal para el desarrollo de la petrolización desplazó al sector primario, que pasó de $11.7 \%$ del total en 1977 a sólo 2.2\% en 1982. Por el contrario, la inversión destinada a la industria petrolera aumentó en el mismo lapso de $68.4 \%$ a $93.2 \%$. Los productos tradicionales como el cacao, el plátano y el coco apenas crecieron y la caña de azúcar disminuyó a la mitad de su producción original entre 1970 y 1983, y hoy en día la producción de estos cultivos está en franca decadencia. El grueso de las participaciones federales para el desarrollo petrolero pasó de $16.310,000$ pesos a 80.981,000 entre 1970 y 1985 y, consecuentemente, la inversión para reactivar el campo se fue desplazando a segundo término (Pinkus y Contreras, 2012: 64-68).

Undocumento reproducido por Roberto Thompson (S/f) fue enviado por la población ejidal de El Carmen al presidente José López Portillo. En él se presentan los argumentos y demandas que hacen las sociedades rurales debido a los daños causados por PEMEX al entorno natural, a sus viviendas y a las actividades 
productivas, tal como se expresa en las siguientes líneas:

Nuestro ejido que consta de 702-00-00 hectáreas ha sido totalmente destrozado por dicha empresa [PEMEX], localizando pozos, caminos, zanjas para meter tuberías y construyendo edificios de diferentes tamaños. Las aguas como fuente de abastecimiento para nuestros animales y nosotros mismos, actualmente se encuentran totalmente contaminadas con capas de aceite y residuos de los mechones que arden día y noche. La lámina de nuestras casas habitación, las cercas de alambre de nuestros potreros y los pastizales, todo se termina en poco tiempo; se nos muere el ganado [...] Caminamos de un lado a otro haciendo saber a diferentes autoridades tanto agrarias como de la empresa Petróleos Mexicanos, para que se nos liquide o se nos indemnice todos los daños que han causado a nuestros intereses (Thompson, S/f apud Martínez, 1996).

La empresa Petróleos de México es cierto que es una gran [empresa que explota la] riqueza [petrolera] de nuestro país, pero únicamente para beneficiar a determinadas personas, nosotros los campesinos siempre hemos sido la parte baja, en donde repercute la humillación y la pobreza y con esto que antes mencionamos queda de manifiesto una vez más nuestra situación económica que cada día que pasa nos hacemos más pobres (Thompson, S/f apud Martínez, 1996).

Para los afectados, este tipo de reclamos escritos no cancelaban otras acciones de resistencia. Así, el 19 de enero 1981 pusieron en práctica lo que sería probablemente el momento más significativo de la estrategia de defensa de su patrimonio: el bloqueo de los campos de producción petrolera. Millares de campesinos participaron para llamar la atención del gobierno federal y para exigir de manera más determinante a la empresa el pago de las indemnizaciones. Entre las acciones de protesta que emprendieron estuvieron: obstaculizar el paso del Puente Limón en la zona fronteriza con Chiapas; la carretera de Reforma a la Estación Juárez hasta llegar a bloquear el Complejo Petroquímico de Cactus; el área industrial de Reforma; tomar 23 equipos de perforación, cuatro de reparación de pozos, nueve baterías, cinco estaciones de compresión y dos plantas de inyección de agua, al mismo tiempo que impidieron la entrada y salida de personal de la paraestatal.

PEMEX denunció los hechos por tratarse de una serie de acciones que eran consideradas ilegales. Sin embargo, los afectados mostraron los expedientes de las reclamaciones que venían haciendo a la empresa desde hacía ya varios años sin obtener solución. La presencia de la fuerza militar estuvo a punto de generar más problemas, pero el gobernador Enrique González Pedrero escuchó las demandas y prometió realizar los pagos a más tardar en un mes. Esta promesa frenó las acciones de resistencia y los demandantes se retiraron con la esperanza de recibir sus respectivas indemnizaciones, las ucales nunca llegaron a tener entre sus manos. ${ }^{4}$

De cualquier manera, esta resistencia social de los pobladores no restó la importancia económica que el petróleo ha tenido para Tabasco y para el país. Entre 1973 y 1992 se produjeron alrededor de 4,864 millones de barriles en esta región, con un promedio de extracción de 666,403 barriles diarios, lo que generó en ese lapso una riqueza de más de 130,000 millones de dólares, cifra superior al monto de la deuda externa que existía al finalizar el régimen presidencial de Carlos Salinas de Gortari.

Por otra parte, los Gobiernos federal y estatal emprendieron grandes obras de drenaje y desecación de terrenos con la finalidad de introducir los ductos y toda la infraestructura indispensable para la explotación y traslado del crudo. Estas obras provocaron grandes cambios en el ambiente natural ya que se afectaron tierras de cultivo y cría de ganado para poner en su lugar las instalaciones de la explotación petrolera. Hubo 
derrames de petróleo crudo, sales, aceites, emulsiones, contaminación atmosférica, entre otros múltiples daños al medio ambiente (Pinkus y Contreras, 2012).

Podemos decir que la intervención de las empresas petroleras en la entidad tabasqueña dio inicio a una nueva fase de deterioro de los paisajes naturales, al mismo tiempo que generó una monetarización de la economía y de la vida cotidiana de los habitantes de la entidad. Este proceso económico repercutió en el alza de los precios de los productos alimenticios, la vivienda, la educación y, en general, el alza de costos en todos los aspectos de la vida a que estaban acostumbrados los habitantes de Tabasco.

Podemos señalar tres grandes causas de depredación del medio ambiente durante el siglo XX: el monocultivo de la producción platanera, la ganadería extensiva y el petróleo ya que todos en su conjunto ocasionaron la deforestación de grandes extensiones de terrenos (Flores, 2006: 15).

A partir del auge de la explotación petrolera en Tabasco la vida de los pueblos mayas y mestizos tabasqueños cambió drásticamente al convertirse sus miembros en población económicamente activa, en fuerza de trabajo barata de los pozos petrolíferos, transformación que significó a su vez la desestructuración de la vida comunitaria, ya que, como bien señala Flores:

... en algunos lugares la población chontal fue prácticamentearrancadadesusactividadestradicionales para ser ocupada en abrir zanjas de oleoductos, como peones y cargadores... (Flores, 2006: 16).

En este sentido, se puede afirmar que las sociedades mayas chontales asentadas en estas comunidades comenzaron a perder numerosos elementos simbólicos de su identidad cultural, entre ellos, el abandono de sus actividades de subsistencia. Al mismo tiempo comenzaron a asumir costumbres, actitudes, acciones y nuevos estilos de vida que fueron introducidos a partir de la explotación petrolera. Desde esta perspectiva, estas sociedades originarias, a diferencia de los mayas de Yucatán y de los mixes o los zapotecos de la sierra de Oaxaca, fueron paulatinamente asimilados al proceso secularizador que propaga la cultura global y que exalta el estilo de vida occidental como meta a alcanzar bajo la premisa del aparente desarrollo que tendrían el estado y las comunidades con la explotación del crudo.

Para el gobierno estatal y las comunidades, era impensable que la industria petrolera, que implica inéditos ingresos para la economía del país, no se tradujera también en mejoras económicas para ellos, por lo tanto, ya no consideraban rentable seguir pensando en aquellas formas de "organización tradicional campesina", basadas en la producción para el autoconsumo, la organización familiar, el pequeño comercio, la cooperación y la ayuda mutua -mano vuelta o tequios-del grupo doméstico y la comunidad de pertenencia.

Hasta poco antes del desarrollo de la industria petrolera, los chontales y otras étnias mayenses o mestizas seguían siendo agricultores, pescadores e incluso ganaderos. No obstante, a raíz de la actividad petrolera y del empobrecimiento del campo, se vieron obligados a migrar paulatinamente hacia los centros urbanos para emplearse como mano de obra en la industria de la construcción: como vendedores ambulantes, o bien como obreros y empleados del sector servicios. Actividades económicas que, según apunta Flores, antes sólo jugaban un papel complementario de la economía campesina y en la actualidad constituyen las principales actividades en torno a las cuales gira la reproducción de estas comunidades (Flores, 2006).

\section{Estudio de caso: Cárdenas, Tabasco}

Cárdenas se localiza en la región de La Chontalpa. Esta ciudad es la cabecera municipal. Se encuentra 
ubicada en los paralelos $17^{\circ} 59^{\prime}$ latitud norte y $91^{\circ} 32^{\prime}$ de longitud oeste. Al norte colinda con el Golfo de México y los municipios de Paraíso y Comalcalco; al sur con el estado de Chiapas y Huimanguillo; al este con los municipios de Comalcalco, Cunduacán y el estado de Chiapas; al oeste con el municipio de Huimanguillo y el estado de Veracruz. Tiene una extensión territorial de 2,112 $\mathrm{km}^{2}$, que representa $8.3 \%$ del territorio total del estado, y ocupa el quinto lugar en la escala de extensión municipal. Su división territorial está conformada por: una ciudad, dos villas, 20 pueblos, 27 rancherías, 65 ejidos, 40 colonias urbanas, cuatro fraccionamientos, seis congregaciones y 20 colonias agrícolas y ganaderas. ${ }^{5}$

Según los resultados preliminares del Censo General de Población y vivienda 2010 del INEGI, el municipio cuenta con 248,507 habitantes, de los cuales 122,280 son hombres y 126,227 son mujeres, lo que representa $11.1 \%$ de la población del estado y el segundo municipio más poblado de Tabasco después del municipio del Centro. La densidad de población del municipio es de 121.3 habitantes por $\mathrm{km}^{2}{ }^{6}$

Dentro de ella existe una población indígena de 306 habitantes. ${ }^{7}$ En el municipio se ubican 25 centros de desarrollo regional en los que se concentran las principales actividades económicas y sociales y donde acuden los habitantes de las poblaciones circunvecinas para satisfacer diversas necesidades, tales como la educación, el comercio, el intercambio y la gestión pública (ver mapa 3). ${ }^{8}$

\section{Clima e hidrografía}

El tipo de clima del municipio es cálido y húmedo, con abundantes lluvias en verano (AM), la temperatura del año más fría es de $25.6^{\circ} \mathrm{C}$, y en los meses más calurosos se alcanza una temperatura de $44^{\circ} \mathrm{C}$. Su temperatura media anual es de $26^{\circ} \mathrm{C}$, con una máxima media mensual de $30.3^{\circ} \mathrm{C}$ en mayo y una mínima media mensual de $20^{\circ} \mathrm{C}$ en diciembre y enero. ${ }^{9} \mathrm{La}$ precipitación pluvial total anual en promedio es de
2,003.2 mm y para el mes más lluvioso es de 2,938.5 mm (Cuaderno Estadístico Municipal, 2004).

El municipio se encuentra en la región del río Mezcalapa que surca su parte oriental. En su territorio se localizan dos albuferas, la Machona y la Del Carmen, las cuales se unen a la laguna de El Pajonal y al Golfo de México por la Barra de Santa Ana. Además se localizan las lagunas de La Palma, Santa Teresa y otras de menor importancia.

En la parte norte se hallan los ríos San Felipe y Naranjeño, que desembocan en la laguna del Carmen, y el río Santana, en la laguna de la Machona. Cerca de la boca del río Tonalá, límite con Veracruz, desemboca el río Chicozapote, que nace en El Lodazal y rodea la villa Benito Juárez. Existen otros ríos y arroyos de menor importancia. ${ }^{10}$

\section{Principales ecosistemas}

La región de Cárdenas cuenta con una diversidad de ecosistemas. Así, la vegetación original es de selva media y alta perennifolia, y la vegetación secundaria la constituyen los cultivos agrícolas, los pastizales y los acahuales. Existe también vegetación hidrófila conocida como popal cuya presencia se debe a las deficiencias de drenajes de los terrenos y a las tierras inundables. Es importante mencionar que esta planta es aprovechada para el tejido de la cestería, siendo una actividad complementaria en su economía. Asimismo, cuenta con árboles maderables que se utilizan en la elaboración de muebles para el uso y venta.

Con respecto a la fauna es posible encontrar reptiles y aves, tales como la iguana (Iguana iguana), especie enlistada en la NOM-059-SEMARNAT-2001 en una categoría sujeta a protección especial (Pr), toloque (Basiliscus vittatus), paloma (Columba spp), pijul (Crotophaga sulcirostris), zopilote (Coragypatratus), entre otros.

Es importante mencionar que toda esta diversidad ecosistémica de Cárdenas ha sido superada por la 
expansión de áreas para pastizales a costa del desmonte y la deforestación con sus resultados consecuentes (ver mapas 6 y 7). Le siguen en importancia el cultivo de caña, cacao y fragmentos de acahuales, que en su mayoría fueron diezmados entre 1976 y el 2000 ante el aumento de la actividad ganadera, lo cual no solamente ha implicado el aumento de potreros para el ganado, sino también el abandono de ciertas actividades de subsistencia como la milpa y otros monocultivos que habían sido introducidos. De esta manera se puede identificar que la mayor parte de la superficie de cada comunidad está destinada al uso agropecuario y una menor superficie a los cultivos de subsistencia y comerciales como el cacao, sorgo, arroz, naranja y plátano."

Entre 1976 y el 2000 del siglo XX, a este rico ecosistema se sumó el creado para la explotación petrolera por parte de PEMEX, a partir de la perforación de los yacimientos para la extracción del crudo. Actualmente hay 169 pozos petroleros en explotación ${ }^{12}$ en 13 campos: Cárdenas (21), Chipilín (1), Edén (6), Jacinto (8), Jolote (10), Mora (9), Otates (6), Rodador (12), San Ramón (22), Sánchez Magallanes (64), Santuario (9) y Tepeyil (1). Se producen 113,507 barriles diarios de petróleo crudo y 222.6 millones de pies cúbicos de gas natural.

Contrastando ambas imágenes para detectar los cambios que ha padecido el uso del suelo durante un lapso de 24 años dentro del municipio de Cárdenas, se puede observar claramente que se ha dado una conversión en los diferentes parches de hábitat existentes. Primero se aprecia que donde se encuentra la cabecera municipal se presenta un manchón que corresponde a la zona urbana, anteriormente ocupada por la agricultura de temporal. Asimismo, se puede observar un incremento de la zona de pastizales, dedicados a la ganadería extensiva en todas direcciones, a costa de diferentes ecosistemas como los tulares, los manglares y agroecosistemas, tal como puede notarse en el noroeste del municipio donde se cambiaron terrenos de temporal por terrenos para pastizales. Además, es posible distinguir una reducción y aislamiento de asociaciones vegetales como el palmar y la selva alta perennifolia, quedando estas superficies inmersas en un área matriz principalmente ganadera, con sus potenciales efectos a nivel ecológico de degradación, ya que impide el flujo e intercambio de organismos que utilizan diferentes nichos del mismo hábitat, con lo que se disminuye la conectividad y, por ende, se reducen las poblaciones de organismos y las cadenas bióticas endémicas de la región. ${ }^{13}$

\section{El auge petrolero y sus consecuencias}

El auge petrolero tuvo dos grandes consecuencias; por una parte, se propaló entre las poblaciones la creencia en la abundancia a partir de una falsa construcción de la imagen de progreso expresada en obras deíndole urbana que beneficiaron fundamentalmente a la capital del estado, Villahermosa, sin que influyeran directamente en las condiciones de vida de las poblaciones rurales. Por otra parte, el desarrollo petrolero originó el surgimiento de una nueva cultura cuyas bases se fincaron en la petrolización de la economía y en la esperanza de mejorar el bienestar en las condiciones de vida de los pobladores, quienes también fueron cambiando paulatinamente sus percepciones respecto a sí mismos, a su identidad y al eje de su economía tradicional, es decir, de sus actividades productivas de subsistencia.

Dos décadas más tarde, las esperanzas de estas sociedades se vieron frustradas al percatarse de que los beneficios de la petrolización de la economía traían consigo pocos impactos favorables en sus condiciones de vida y, por el contrario, estaban ocasionando directamente el deterioro de sus recursos naturales, de sus propias actividades productivas, así como la de sus viviendas y la cría de sus animales de traspatio. Todo ello como efecto de la alta contaminación que provocaba la extracción del crudo, del gas, el uso de diversos químicos, así como los constantes derrames y la polución que generaban estos últimos (ver foto 1$).{ }^{14}$ 
En septiembre de 1976, como consecuencia del desarrollo desigual en las zonas petroleras de Tabasco, surgió un movimiento social que se autodesignó Pacto Ribereño, para oponer resistencia a la arrolladora presencia de PEMEX y a los efectos nocivos que ocasionaba en las economías familiares, en la salud de los habitantes y en todo el entorno natural. Los líderes e integrantes de estas movilizaciones tuvieron como objetivo la exigencia de indemnizaciones por parte de PEMEX para resarcir la destrucción de las tierras ejidales y las pequeñas propiedades, y revertir los daños a las propiedades de los finqueros, rancherías y lagunas, por la alta contaminación que padecían y que estaba afectando el sistema ecológico del sureste, impactos que también incidían sobre la población, en especial en la economía sobre la que fincaban su reproducción social (Velázquez, 1981; Martínez, 1996).

Como bien señalaban los grupos de resistencia, las consecuencias ambientales que generó PEMEX fueron desastrosas ya que la construcción de los gasoductos y petroductos, además de las represas, originó el desvío de los cauces de los ríos, con lo cual algunas lagunas se volvieron más profundas por el dragado y otras se sedimentaron o se secaron. De igual manera fue afectada la actividad pesquera, no sólo por el desvío de las aguas, sino porque se asentaron en ellas metales pesados que afectaron la fauna acuática y demás animales cuyo nicho ecológico se halla en la ribera de los cuerpos de agua y la vegetación circundante, debido a la salinización que originó la explotación del petróleo en la región (Velázquez, 1981; Martínez, 1996).

Pese a la lucha emprendida por los grupos de resistencia, estos no obtuvieron el resultado esperado (la indemnización) por diversos motivos, entre ellos que los líderes se corrompieran y fueran cooptados por la paraestatal petrolera. Además, PEMEX recurrió a los amparos legales que prolongaron el tiempo de la lucha, hasta que ésta se diluyó y así la paraestatal evitó pagar las indemnizaciones que los campesinos afectados y no afectados le exigían (ver foto 2 y foto 3 ). ${ }^{15}$
Comosehavistoyaampliamentelastransformaciones medioambientales, económicas, sociales, políticas y culturales en las cuatro comunidades estudiadas del municipio de Cárdenas, Tabasco, fueron resultado de tres grandes procesos de desarrollo en el transcurso del siglo XX, los cuales fueron causa de la depredación ambiental que actualmente padece este municipio y toda la entidad. En términos de estos daños al ecosistema y del deterioro de las condiciones de vida de amplios sectores de la población, el costo de las políticas de desarrollo que se impulsaron en Tabasco, sobre todo a partir de 1960, fue muy elevado y, como bien apunta Tudela, este proceso: “[...] de ninguna manera podría considerarse como inevitable [...]".

Las estrategias productivas que se llevaron a cabo en Tabasco durante el siglo XX siempre aludieron a la necesidad de incorporar la región al "desarrollo económico". En algunos casos se intentó explotar algún recurso natural local con criterios inmediatistas y productivistas a través de la inserción de algún proceso productivo cuya integración regional fue, la mayoría de las veces, escasa o deficiente. En otros casos se adoptó una estrategia basada en una intensificación productiva y la incorporación de tecnologías "modernas" sin tener conciencia del impacto socioambiental que se iba a ocasionar. En el sentido más estricto, la "modernización" se refiere a este tipo de estrategia productiva. Según este autor, la "Tradición y modernización constituyen los dos polos de un falso dilema, en la medida en que ambas, en la forma concreta que asumieron en la historia regional, han conducido a situaciones semejantes de deterioro" (Tudela, 1996: 439-440).

Respecto a las cuatro comunidades analizadas en este trabajo, José Ma. Pino Suárez C-22; Veinte de Noviembre C-33; Ing. Eduardo Chávez Ramírez C-27 y la Colonia Ingenio Benito Juárez, ${ }^{16}$ cabe señalar que, si bien en ellas PEMEX emprendió la perforación de pozos para la extracción del crudo, desde los cincuenta y setenta la actividad de extracción no se realizó y los pozos quedaron en reserva. Hoy en día la paraestatal ha 
retomado el proyecto de explotación de dichos pozos, situación ante la cual los pobladores han generado toda una serie de expectativas, las cuales se fundamentan en las experiencias que han observado a partir de los procesos acontecidos en Cárdenas y otros municipios, donde el común denominador ha sido el deterioro de los diversos ámbitos de la vida y del entorno natural de dichas comunidades.

Sin embargo, es cierto que en estos poblados se ha dado un mínimo desarrollo económico en términos de una mayor circulación del efectivo temporal, que ha posibilitado a un sector muy específico de la población tener un mayor poder adquisitivo para obtener algunos bienes muebles e inmuebles. También se puede aseverar que los datos de las encuestas realizadas a los jefes de familia de los poblados demuestran que, del otro lado de la balanza, existe hoy en día una población económicamente pauperizada, la cual ha tenido que abandonar no sólo su entorno natural y sus actividades productivas rurales, sino también migrar a las ciudades y otras regiones del país e incluso a los Estados Unidos en busca de trabajo y mejores condiciones de vida para ellos y sus familias (Pinkus y Contreras, 2012).

Las comunidades con promesas de altos grados de desarrollo se encuentran hoy en franca decadencia. La poca infraestructura interna con que contaban ha quedado tan obsoleta que hoy en día se encuentran sin incentivos para la producción. Esta situación se ve agravada por el constante robo de sus pocas cosechas y animales de crianza, lo cual afecta a muchos de sus pobladores, hombres y mujeres en edad productiva, que ya están viciados y se dedican a delinquir ante la desesperanza y la miseria en que viven.

Para concretar estas maneras de concebir y crear las expectativas que les ha generado la industria petrolera, los habitantes de estas poblaciones, no obstante que aún no se ha llevado a cabo de lleno la explotación petrolera, han asumido como estrategia su organización para iniciar reclamos sobre los supuestos daños que PEMEX generará en sus comunidades. En este sentido, los pobladores de estas comunidades, con base en las experiencias de los pueblos vecinos, han asumido la "cultura de la reclamación", ya que, según señalaron los informantes de las comunidades en estudio, han comenzado a sumarse a las organizaciones que han asumido la lucha por el bienestar del medioambiente y de las familias campesinas (ver foto 4 ).

Dirigentes campesinos y pescadores de los municipios de Huimanguillo, Cárdenas, Cunduacán, Jalpa deMéndez y Comalcalco, pusieron un plazo [...] para que PEMEX pague los daños que ha ocasionado. El líder campesino sometió a consideración de los presentes si estaban dispuestos a retomar esta lucha. Todos aceptaron. ${ }^{17}$

Con base en los 200 cuestionarios levantados podemos señalar que, ante este panorama, $65 \%$ de los pobladores (jefes de familia) de las cuatro comunidades, manifestaron tener una percepción generalizada del significado negativo que puede tener el desarrollo de la explotación petrolera, sobre todo en cuanto al aspecto de la contaminación y destrucción que producen sus actividades. De hecho, la mayoría de los habitantes de estas comunidades, a pesar de que no han sido impactados directamente por la explotación petrolera, nos aseguraron que ya habían sido afectados por las actividades de la paraestatal, ya que la contaminación ambiental ocasionada por los derrames y emanaciones les ha llegado desde varios kilómetros hasta sus poblaciones. Cabe señalar que, pese a estas aseveraciones de los entrevistados, pudimos constatar, con base en el levantamiento de encuestas en "Tiempo Cero" que realizaron los biólogos, investigadores de la Universidad Juárez Autónoma de Tabasco durante el 2008 y 2009, ${ }^{18}$ y en las pruebas aplicadas en los suelos, cuerpos de agua y vegetación de los poblados ya mencionados y seleccionados para el estudio, no se detectaron rastros de elementos contaminantes provenientes del petróleo y sus derivados. 
De la anterior confrontación entre la percepción de la abundancia que se propaga en estas comunidades y los impactos que genera la industria petrolera, podemos deducir que las demandas que actualmente están haciendo los líderes rurales de las comunidades estudiadas, están asociadas con el interés de verse beneficiados por los proyectos productivos que la paraestatal prevé impulsar entre los campesinos para que tengan otras alternativas económicas, para evitar la resistencia a la expansión petrolera en estas comunidades y para disminuir el número de reclamaciones que les han implicado costosas indemnizaciones. Pero también es ineludible reconocer que los campesinos perciben en esta lucha de intereses la posibilidad de verse beneficiados con las compensaciones que la empresa paraestatal se ha visto obligada a entregar a los afectados para lograr su cometido de expandirse en aquellos pozos que se encontraban en reserva.

Asimismo, resulta evidente que, dentro de esta aparente percepción negativa de las comunidades entrevistadas, los pobladores guardan la esperanza del atractivo económico que representa la entrada de PEMEX a sus comunidades, expectativas que en definitiva son positivas para ellos en términos de la derrama económica o supuestos empleos que la actividad puede introducir en las economías locales y en los grupos familiares.

En efecto, a partir de las encuestas, resultó que $65 \%$ de las personas declaró que aceptaría que PEMEX realice trabajos en sus comunidades; $79 \%$ de los encuestados se mostró interesado en recibir información de la paraestatal, con la finalidad de enterarse de los beneficios que obtendría a partir de la introducción de la actividad; y 86\% señaló abiertamente su interés por que PEMEX entrara en su comunidad, siempre y cuando sus miembros estuvieran involucrados en las actividades que se desarrollarían, ya que ello traería grandes beneficios a su comunidad.

\section{Conclusiones}

En resumen, a partir del siglo Xx, la población tabasqueña y particularmente las comunidades rurales, comenzaron a experimentar transformaciones fundamentales en su organización social: en la estructura familiar, en sus formas de producción y, en general, en sus relaciones con el medio ambiente, así como en la forma de regir la vida política. También hubo cambios en el vestido, el uso del idioma y en sus creencias religiosas, ya que, según los informantes encuestados, la mayoría de las mujeres originarias de la región comenzaron a cambiar su indumentaria por la ropa occidental, asimismo, dejaron de hablar su lengua nativa y a comunicarse con mayor frecuencia en español, debido al mayor contacto y presencia del personal que PEMEX envió a las comunidades a trabajar en la industria extractiva; de manera paralela al abandono de las practicas agropecuarias de subsistencia, las comunidades comenzaron a dejar de realizar los rituales acostumbrados paulatinamente.

Evidentemente, estos cambios estuvieron relacionados con los principales procesos de desarrollo socioeconómico en el estado de Tabasco como: los programas de diversificación y modernización agropecuaria, y la actividad petrolera, por mencionar los dos más importantes, que generaron diversos impactos negativos en las poblaciones, como la migración, la deforestación y, consecuentemente, en la organización de la sociedad rural tabasqueña.

En cuanto al costo ambiental, la modernización tecnológica, el desarrollo económico, así como otras políticas públicas neoliberales impulsadas por el Estado, tuvieron una fuerte incidencia en la dinámica del medio biofísico, cuyos impactos experimentados fueron más dañinos, intensos e irreversibles durante los últimos sesenta años, que el deterioro que había sufrido el medioambiente natural en los cinco siglos anteriores. En términos ecológicos, este deterioro manifestó sus consecuencias en el potencial productivo regional $y$, 
por consiguiente, en las perspectivas de bienestar de la población local (Tudela, 1992: 365-366).

De acuerdo con lo señalado por Tudela podemos decir que los factores desencadenantes de los cambios en la hidrología de la planicie costera del Golfo sur son: la deforestación, el desarrollo de la infraestructura hidráulica y la construcción y apertura de las comunicaciones terrestres. De hecho, la tala intensiva de la selva endémica fue un proceso común para toda la región que, junto con los cambios hidrológicos, modificaron el cauce de los ríos y conllevaron afectaciones que disminuyeron la capacidad para retener los escurrimientos de las tres cuencas regionales.

Por su parte, la intervención hidráulica fue ejercida en forma diferencial, ya que la cuenca "GrijalvaVillahermosa" recibió un intenso impulso a través del represamiento de la cuenca alta y por las obras de contención del cauce principal. Del mismo modo se intervino en la cuenca "Tonalá y Lagunas del Carmen y Machona”, particularmente en el drenaje superficial de La Chontalpa y en las obras de infraestructura carretera.

Todas estas intervenciones tecnológicas en el manejo de la red hidrológica de la planicie tuvieron un efecto directo en las lagunas costeras a través del incremento del azolve, que provocó una disminución de la fauna estuarina y la capacidad de las cuencas para retener las aguas, ya que impiden los flujos entre los cauces de los ríos, los pantanos, las lagunas y las llanuras de inundación y dirigen a mayor velocidad los escurrimientos hacia el mar, ocasionando inundaciones de las zonas pobladas. De igual forma, el proceso de erosión-transporte-depositación que se llevaba a cabo en toda la red hidrológica de la cuenca se alteró: se incrementó la erosión y la depositación, y ahora se dificulta el transporte de sedimentos (Tudela, 1989: 365-366).

En síntesis, el sistema socioambiental de Tabasco ha sufrido en el último siglo cambios originados por la puesta en marcha de políticas de desarrollo económico sin precedentes en la región. Como vimos anteriormente, las transformaciones sufridas en el sistema social y natural obedecen principalmente a los siguientes grandes procesos socioeconómicos:

a. La modernización e intensificación agrícola, desarrollada en base a un patrón tecnológico similar al que se había utilizado con anterioridad en las zonas semiáridas del país, implicó la dependencia hacia los insumos para la producción y subsidios de las dependencias públicas. Asimismo, este proceso exigió el desmonte y la desecación de amplias extensiones de tierras húmedas.

b. Laganadería extensiva, aunque poco tecnificada, fue un proceso que se desencadenó por la disponibilidad de infraestructura de transporte terrestre, la cual fue la principal responsable del desmonte de extensas áreas selváticas regionales para ser sustituidas por pastizales.

c. La intensificación de la industria petrolera se colocó por encima de las dos anteriores actividades de desarrollo económico precipitando la crisis de éstas, cuyas condiciones estructurales, comparadas con la tecnología de la actividad petrolera, estaban obsoletas.

Contrariamente a lo que se esperaba de dichos proyectos de desarrollo económico, en la actualidad el sistema socioambiental tabasqueño refleja un potencial de desarrollo regional bastante disminuido en relación con el que existía hace más de setenta años como resultado del deterioro ambiental que se ha producido en términos de la cantidad de biomasa, producción primaria, diversidad ecológica, reserva de germoplasma y fertilidad de los suelos (Tudela, 1989: 441-443).

Si bien el sistema ya ha sido impactado con grandes alteraciones ambientales, también es cierto que los nuevos ecosistemas dominantes, como los pastizales, siguen siendo menos productivos que las selvas, aunque 
resultan ser bastante estables desde el punto de vista ecológico. De cualquier modo, los procesos ambientales desencadenados por la intervención humana tienen su propia dinámica y algunos pueden seguir actuando una vez se estabilicen las acciones que les dieron origen (Pinkus, 2010).

Por último es importante señalar que, en el contexto actual, la población rural de Tabasco, sus indígenas y sus descendientes, a más de medio siglo de haberse iniciado los programas de diversificación productiva, siguen siendo marginados por la sociedad dominante. Si bien en el discurso oficial siempre se ha manifestado una preocupación por su "progreso", en realidad se promueve un modelo de desarrollo contrario a su ideología, modo de vida y entorno natural. Muestra de ello han sido los constantes proyectos de desarrollo regional que han terminado en fracasos y sin distribución equitativa de los recursos ejercidos. No obstante, los movimientos sociales que desencadenaron la inserción del proceso petrolero en la región se han desactivado. Esto no significa que Tabasco haya logrado la estabilidad social y que obedezca a una sensibilidad política de la sociedad originaria ocasionada por la disponibilidad de recursos económicos provenientes de la explotación petrolera, sino más bien que la inminente necesidad de sobrevivencia de los habitantes de los poblados los obliga a enviar su fuerza de trabajo en edad económicamente activa en busca de fuentes de trabajo e ingresos, a las ciudades de la entidad de donde incluso se los expulsa obligándoles a migrar a otros lugares y países en busca de la manutención propia y de sus familias; hecho primordial que los distrae de sus capacidades de organización y lucha por una reproducción social más justa, humana y respetuosa de su entorno natural que es fuente de viday de preservación de la sociedad en este planeta.

\section{Notas}

${ }^{1}$ La percepción de la gente ha sido utilizada en los últimos años como herramienta medular para valorar y regular el impacto más importante en los problemas ambientales. Ésta también ha servido para implementar y redireccionar los programas de gobierno respecto al uso, control y acceso de los recursos naturales. La base de estas acciones son la participación social y el interés colectivo de la comunidad. El fin y principal objetivo de este proyecto socioambiental es incrementar la calidad de vida de los actores sociales y disminuir el impacto real y el percibido.

2 Estas facultades fueron dadas en el decreto de conformación de la Comisión del río Grijalva en 1951 y fueron ratificadas posteriormente para la Chontalpa en 1965.

3 "Durante estas dos décadas la orientación de la industria petrolera sería la misma: satisfacer las necesidades internas con precios subsidiados, como forma de promover el crecimiento industrial del país" (Flores, 2006: 14).

${ }^{4}$ Según Martínez (1996), la cuantificación de los supuestos daños realizada por el Pacto Ribereño seguía siendo la misma cuando el gobierno local realizó esfuerzos para llegar a una negociación con PEMEX; pues se supone que en 1982 la empresa pagó 2,050 millones de pesos inicialmente y el resto después, pero problemas de la organización impidieron que las indemnizaciones llegaran directamente a los afectados. Ignorantes de que la empresa había hecho las liquidaciones, los campesinos pidieron la renuncia de su asesor jurídico, el licenciado Arturo Acosta Vega. Eulogio Méndez Pérez, presidente del Pacto y miembro de la Confederación Nacional Campesina (CNC), declaró que cuando se percató en junio de que habían sido defraudados, le hicieron una oferta de siete millones de pesos por su silencio. Nadie supo a ciencia cierta cuál fue el destino del monto supuestamente pagado por PEMEX, pero los miembros del Pacto insistieron en que no lo recibieron.

${ }^{5}$ Municipio de Cárdenas (Tabasco), autor, colaboradores de Wikipedia, editor Wikipedia: La enciclopedia libre (2012, 23 de junio); Fecha de consulta: 27 de junio de 
2012, desde URL Permanente: http://es.wikipedia.org/ wiki/Municipio_de_Cárdenas_(Tabasco).

${ }^{6}$ Municipio de Cárdenas (Tabasco), autor, colaboradores de Wikipedia, editor Wikipedia: La enciclopedia libre (2012,23 de junio); Fecha de consulta, 27 de junio de 2012, desde URL Permanente: http://es.wikipedia.org/ wiki/Municipio_de_Cárdenas_(Tabasco).

${ }^{7}$ El II Conteo de Población y Vivienda del 2005 señala que todavía habitan un total de 820 personas que hablan alguna lengua indígena. De acuerdo a este conteo 67 hablan náhuatl, 58 maya, 57 zapoteco, 50 chontal de Tabasco y el resto lo componen otros grupos sin clasificación definida.

${ }^{8}$ Estos centros son villa Andrés Sánchez Magallanes, Villa Benito Juárez, poblado Ignacio Gutiérrez Gómez, poblado C-23 (Venustiano Carranza), poblado C-1l (José María Morelos y Pavón.), poblado Azucena 2a, poblado El Golpe, ranchería. Santuario 2aㅡ, poblado C-10 (Lázaro Cárdenas), poblado C-9 (Francisco I. Madero), poblado C-14 (Plutarco Elías Calles), poblado C-15 (Adolfo López Mateos), poblado C-16 (Emiliano Zapata), poblado C-17 (Independencia), poblado C-22 (José María. Pino Suárez), poblado C-2l(Benito Juárez), poblado Santana la ${ }^{a}$, poblado Santa Rosalía, r/a. Hidalgo $2^{\underline{a}}$ B, poblado C-27 (Eduardo Chávez), poblado C-28 (Gregorio Méndez), poblado C-33 (20 de Noviembre), poblado Melchor Ocampo, poblado Habanero $1^{\underline{a}}$ y poblado El Barí, del municipio de Cárdenas (Tabasco), autor, colaboradores de Wikipedia, editor Wikipedia: Laenciclopedia libre (2012,23 de junio); Fecha de consulta, 27 de junio de 2012, desde URL Permanente: http:// es.wikipedia.org/wiki/Municipio_de_Cárdenas (Tabasco).

${ }^{9}$ Bueno, Álvarezy Santiago (2005); Pinkus Rendón(2010); Municipio de Cárdenas (Tabasco), autor, colaboradores de Wikipedia, editor Wikipedia: La enciclopedia libre (23 de junio de 2012), consulta: 27 de junio de 2012, desde URL Permanente: http://es.wikipedia.org/wiki/ Municipio_de_Cárdenas_(Tabasco).

${ }^{10}$ Ver nota 9.
${ }^{11}$ Ver nota 9.

12 Total 13 campos: Cárdenas (21), Chipilín (1), Edén (6), Jacinto (8), Jolote (10), Mora (9), Otates (6), Rodador (12), San Ramón (22), Sánchez Magallanes (64), Santuario (9), y Tepeyil (1). Se producen 113,507 barriles diarios de petróleo crudo, y 222.6 millones de pies cúbicos de gas natural. J. Bueno, F. Álvarez y S. Santiago (eds.) 2005. Biodiversidad del Estado de Tabasco. Instituto de Biología UNAM CONABIO. Pinkus Rendón, Miguel, 2010, "Historia ambiental de las riberas del Usumacinta en Tabasco", Ruz Sosa, Mario H. (edit.), 2010, Paisajes de río, ríos de paisaje. Navegaciones por el Usumacinta, México, UNAM.

${ }^{13}$ Bueno, Álvarez y Santiago (2005); Pinkus Rendón (2010),

${ }^{14}$ Ver Pinkus y Contreras (2012).

${ }^{15}$ Ver Pinkus y Contreras (2012).

${ }_{16}$ Para el análisis de estos cuatro poblados de la Chontalpa partimos de la elaboración de 200 encuestas repartidas proporcionalmente en cada una de las comunidades de acuerdo con su población. Para ello contamos con el apoyo de los alumnos de la división de Ciencias Sociales y Humanidades de la UJAT. Fueron aplicadas en el primer semestre del 2008. Igualmente se hicieron las muestras en tiempo cero por investigadores y becarios de la División de Ciencias Biológicas de la UJAT.

${ }_{17}$ Fuente: Periódico de la sociedad civil La Verdad del Sureste, junio, 2009.

${ }^{18}$ Investigaciones realizadas por investigadores de la División de Ciencias Biológicas.

\section{Bibliografía}

Arias G., M.E., A. Lau J. y X. Sepúlveda O. (comps.) (1987), Tabasco. Textos de su historia, vol. 1, Villahermosa, Tabasco: Instituto de Investigaciones José María Luis Mora/Gobierno del Estado de Tabasco.

Arias Rodríguez, José Manuel e Ireta Guzmán Hugo (2007), Los donativos y donaciones de PEMEX a Tabasco, 
Villahermosa, Tabasco: Centro de Análisis e Investigación Fundar/Asociación Ecológica Santo Tomás, pp. 5-7

Barrera, Marciano (2005), Apuntes sobre los ríos de Usumacinta 1827-1834, México: CNCA/INAHCentro INAH, Campeche/Gobierno del Estado de Campeche.

Beltrán, José Eduardo (1985), Petróleo y desarrollo, México: Villahermosa, CEIS.

Bueno, J., F. Álvarez y S. Santiago (eds.) (2005), Biodiversidad del Estado de Tabasco, México: Instituto de Biología-UNAM/CONABIO.

Berg, Cornelius (1972), "Olmedieae, Brosimeae (moraceae)", en Flora Neotrópica, Monograph, núm. 7.

Canudas Sandoval, Enrique (1993), El fin del dominio español. El caso de los infidentes tabasqueños [manuscrito], México.

Canudas Sandoval, Enrique (1994), Trópico rojo, t. IV, Inquietudes, México: Ediciones y Publicidad.

Centro de Investigaciones y Estudios en Antropología Social, Pacífico Sur (2006a), "Perfil indígena", en Fichas. Pueblo cho'l de Tabasco, http://pacificosur.ciesas. edu.mx/fichas.html.

Centro de Investigaciones y Estudios en Antropología Social, Pacífico Sur (2006b), "Perfil indígena", en Diagnóstico estatal. Tabasco, http://pacificosur. ciesas. edu.mx/diagnosticoestatal.html.

Centro de Investigaciones y Estudios en Antropología Social, Pacífico Sur (2006c), "Perfil indígena", en Chontales de Tabasco, http://pacificosur.ciesas.edu.mx/ perfilindigena.html.

Centro de Investigaciones y Estudios en Antropología Social, Pacífico Sur (2006d), "Perfil indígena", en Fichas. Pueblo tzeltal de Tabasco, http://pacificosur. ciesas.edu.mx/fichas.html.

Centro de Investigaciones y Estudios en Antropología Social, Pacífico Sur (2006e), "Perfil indígena", en Diagnóstico estatal. Chiapas, http://pacificosur.ciesas. edu.mx/diagnosticoestatal.html.
Centro de Investigaciones y Estudios en Antropología Social, Pacífico Sur (s/f), "Plan Puebla Panamá. Documento base Capítulo México", en Estudios y proyectos. Documentos de contexto, www.ciesas-golfo. edu.mx/istmo/docs/pppbase/indice.htm.

Congreso del Estado de Tabasco-LVI Legislatura (1995), Ley que crea la Comisión Interinstitucional para el Medio Ambiente y el Desarrollo Social.

Contreras Sánchez, Alicia del Carmen (1990), Historia de una tintórea olvidada: proceso de explotación del palo de tinte, 1750-1807, México: UADY.

David J. Palma, et al. (1985), Caracterización de los suelos de Tabasco. Uso actual, potencial y taxonomía, México: Gobierno del Estado de Tabasco.

Flores López, José M. (2006), Chontales de Tabasco, México: Comisión Nacional para el Desarrollo de los Pueblos Indígenas (CDI)/Gaceta Parlamentaria, junio 2000.

García Moll, Roberto (2003a), "Tabasco una visión general”, en Arqueología Mexicana, vol. XXI, núm. 6l, pp. 12-17, México: INAH/Editorial Raíces.

García Moll, Roberto (2003b), "Sitios arqueológicos de Tabasco”, en Arqueología Mexicana, vol. XXI, núm. 6l, pp. 72-79, México: INAH/Editorial Raíces.

García Payro, Olinda (1996), Historia y geografía del estado de Tabasco, México: Santillana.

Gobierno del Estado de Tabasco (1994a), Diccionario Enciclopédico de Tabasco, tomo I, Villahermosa, Tabasco: Gobierno del Estado de Tabasco/Instituto de Cultura de Tabasco.

Gobierno del Estado de Tabasco (1994b), "Los Chontales de Nacajuca”, en Compendio Monográfico, México: Gobierno del Estado de Tabasco-Secretaria de Educación, Cultura y Recreación.

Gobierno del Estado de Tabasco (2002), Enciclopedia de los Municipios de México. Tabasco: Información Municipal, México: Gobierno del Estado de Tabasco 20022006.

Gillespie, Andrew, Bocanegra-Ferguson, D. y JiménezOsornio Juan (2004), "The propagation of Ramón (Brosimum alicastrum Sw.; Moraceae) in Mayan 
homegardens of the Yucatan peninsula of Mexico", en New Forrest, núm. 27, pp. 25-38.

Gurría Lacroix, Jorge (1982), Atlas histórico de Tabasco. 1570-1981, México: Universidad Nacional Autónoma de México-Instituto de Investigaciones Históricas/ Gobierno del Estado de Tabasco.

H. Ayuntamiento Constitucional del Municipio de Nacajuca (2001), Compendio Monográfico del Municipio de Nacajuca, México: H. Ayuntamiento Constitucional del Municipio de Nacajuca, Tabasco 2001-2003.

Instituto Nacional de Estadística, Geografía e Información (1972), IX Censo general de población, 1970, México: INEGI.

Instituto Nacional de Estadística, Geografía e Información (1982), X Censo general de población, 1980, México: INEGI.

Instituto Nacional de Estadística, Geografía e Información (1996), División Territorial del Estado de Tabasco de 1810 a 1995, México: INEGI.

Instituto Nacional de Estadística, Geografía Información (1997), Pueblo Nuevo de las Raíces, Surgimiento y Desarrollo de un Ejido Tabasqueño, México: INEGI.

Instituto Nacionalde Estadística, Geografía Información (2000a), Ciudades Capitales: Una Visión Histórica Urbana, disco compacto, vol. 4, México: INEGI.

Instituto Nacional de Estadística, Geografía e Información (2000b), XII Censo General de Población y Vivienda 2000, México: INEGI, http://sc.inegi.gob.mx/ simbad/index.jsp, [consulta: junio 2008].

Instituto Nacional de Estadística, Geografía e Información (2001), Indicadores sociodemográficos de México, 1930-2000, México: INEGI.

Instituto Nacional de Estadística, Geografíae Información (2004), Cuaderno estadístico municipal, México: INEGI.

Instituto Nacional de Estadística, Geografía e Información (2005), Marco Geoestadístico Municipal, México: INEGI.

Instituto Nacional de Estadística, Geografía e Información (2006a), Población total, género -19302005- en la entidad, México: INEGI, http://www. inegi.gob.mx/est/contenidos/espanol/rutinas/ept.asp ?t=mpob9l\&s=est\&c=3837\&e=27, [consulta: 16 de abril de 2008].

Instituto Nacional de Estadística, Geografía e Información (2006b), Crecimiento poblacional-tasa media anual - 1950-2005 - en la entidad, México: INEGI, http:// www.inegi.gob.mx/est/contenidos/espanol/rutinas/ ept.asp?t=mpob94\&s=est\&c=3840\&e=27, [consulta: 26 de abril de 2008].

Instituto Nacional de Estadística, Geografía e Información (2008a), Archivo histórico de localidades, México: INEGI, http://mapserver.inegi.gob.mx/AHL/ activaTiposBusqueda.do, [consulta: 15 de abril de 2008].

Instituto Nacional de Estadística, Geografía e Información (2008b), "X Censo General de Población y Vivienda 1980", en Sistema municipal de base de datos (SIMBAD), México: INEGI, http:// sc.inegi.gob.mx/simbad, [consulta: 30 de abril de 2008].

Instituto Nacional de Estadística, Geografía e Información (2010), Archivo histórico de localidades, México: INEGI, http:// mapserver.inegi.gob.mx/AHL/activaTiposBusqueda.do, [consulta: abril 15 de 2008].

Instituto Nacional de Estadística, Geografía e Información (2011), Conjunto de Datos Geográficos de la Carta Topográfica, México: INEGI.

Isaac-Márquez, R. et al. (2004), Políticas públicas y agricultura campesina: Patrones de uso del suelo $y$ deforestación en el oriente de Tabasco, Avances de Investigación, Villahermosa, Tabasco: El Colegio de la Frontera Sur.

López Camacho, Javier (1988), "Atlas arqueológico de Tabasco", en Fernández Tejedo, I., M. Gaxiola, J. López Camacho y E. Ramírez (eds.), Zonas arqueológicas. Tabasco, México: Instituto Nacional de Antropología e Historia, pp. 66-102.

López Reyes, Diógenes (1980), Historia de Tabasco, México: Consejo Editorial del Gobierno del Estado de Tabasco. 
Lowe, Linneth (2007), $8^{\circ}$ Coloquio de Doctorado en Estudios Mesoamericanos, México: UNAM, (manuscrito).

Martin, Simon (2003), "Moral-Reforma y la contienda por el oriente de Tabasco", en Arqueología Mexicana, vol. XI, número 6l, pp. 44-47.

Martínez Assad, Carlos (1996), Breve historia de Tabasco, México: Fideicomiso Historia de las Américas/ Colegio de México/Fondo de Cultura Económica.

Muñoz Ortiz, Humberto (1964), Gregorio Méndez (El predestinado) 1836-1887. Su vida y su tiempo, Villahermosa, Tabasco: Tipografía Godínez.

Pérez Chan, Armando (2000), Frontera: Legendario Puerto Marítimo y Fluvial de Tabasco. Síntesis Histórica, Villahermosa, Tabasco: Gobierno del Estado de Tabasco.

Pinkus Rendón, Manuel Jesús (2007), Pueblos mayas y mestizos de Tabasco ante la globalización. El Plan Puebla Panamá en Boca del Cerro, en tesis de dctorado en Estudios Mesoamericanos, México: UNAM.

Pinkus Rendón, Manuel Jesús (2010a), Entre la selva y el río. Planes internacionales y políticas públicas en Tabasco: La globalización del Cañón del Usumacinta, Mérida, Yucatán, Universidad Autónoma de Yucatán/Plaza y Valdés.

Pinkus Rendón, Manuel Jesús (2010b), "Pueblos mayas y mestizos de Boca del Cerro, Tenosique, y sus alternativas turísticas", en Ruz Sosa, Mario H. (ed.), Paisajes de río, ríos de paisaje. Navegaciones por el Usumacinta, México: UNAM/Fomix Tabasco.

Pinkus Rendón, Manuel y Contreras Sánchez, Alicia (2012), Desde la modernización al desarrollo petrolero en Tabasco ¿Quimera o realidad? Diagnóstico socioambiental de la zona petrolera de Cárdenas, México: UADY-UJAT, manuscrito.

Pinkus Rendón, Miguel (2010), "Historia ambiental de las riberas del Usumacinta en Tabasco", en Ruz Sosa, Mario H. (ed.), Paisajes de río, ríos de paisaje. Navegaciones por el Usumacinta, México: UNAM/Fomix Tabasco.

Ramírez Carrillo, Luis A. (2007), “Empleo y capacitación en la actividad petrolera de Yucatán. Propuesta regulatoria”, en Torres Góngora, B., En la ruta del petróleo: impacto de una eventual explotación petrolera en Yucatán, tomo IV, México: Fundación Plan Estratégico de Mérida, Yucatán, p.p. 297-345.

Rosado G., Manuel (1962), Historia y geografía de Teapa, México: Gobierno del Estado de Tabasco.

Rovirosa, José Narciso (1980), Nombres Geográficos del Estado de Tabasco, México: Consejo Editorial del Gobierno del Estado de Tabasco.

Ruíz Abreu, Carlos (1989), Comercio y milicias de Tabasco en la Colonia, México: Gobierno del Estado de Tabasco.

Ruz Sosa, Mario Humberto (1994), Historia de los Pueblos Indígenas de México un Rostro Encubierto: Los Indios del Tabasco Colonial, México: CIESAS/INI.

Ruz Sosa, Mario Humberto (2006), Mayas. Pueblos indígenas del México contemporáneo, México: CDI/PNUD.

Salazar Tosca, José C. (comp.) (1990), Compendio Monográfico de la Historia de Tabasco, Villahermosa, Tabasco: Obra Literaria Pedagógica.

Secretaria de Agricultura, Ganadería, Desarrollo Rural, Pesca y Alimentación (2003), Distrito de Desarrollo Rural 192. Plan Balancán-Tenosique, www.sagarpa.gob. $\mathrm{mx} / \mathrm{dlg} / \mathrm{tabasco} / \mathrm{ddr} 5 / \mathrm{ddr} 5 . h t m, \quad[$ consulta: 10 de octubre de 2007].

Secretaría de Economía (1956), Estadísticas Sociales del porfiriato 1877-1910, México: Poder Ejecutivo Federal.

Secretaría de Educación Pública (1988), Historia mínima de Tabasco, México: Gobierno del Estado de Tabasco/ Instituto Nacional para la Educación de los Adultos/ SEP.

Secretaría de Educación Pública (1991), Tabasco. Cálida Planicie, Húmeda Riqueza. Monografía Estatal, México: SEP.

Solano Palacios E. (2009), "Desarrollo sustentable en zonas afectadas por la industria petrolera”, en Ecofronteras, núm 24, México: Colegio de la Frontera Sur.

Suárez Rodríguez, Ángel (1983), Monografía del municipio de Paraíso, Tabasco, Tabasco: Casa de la Cultura Paraíso.

Revista LiminaR. Estudios Sociales y Humanísticos, año 10, vol. X, núm. 2, julio-diciembre de 2012, San Cristóbal de Las Casas, Chiapas, México. ISSN: 1665-8027 
Subsecretaría de Turismo del Gobierno del Estado de Tabasco (2006), Mapa de las Subregiones de Tabasco, México: Gobierno del Estado de Tabasco.

Torruco Saravia, Geney (1976), "Huimanguillo, Tabasco", en Geografía, historia, sociedad y economía de un municipio, México, mimeógrafo de 95 páginas.

Torruco Saravia, Geney (1979), Huimanguillo, Tabasco. Sus límites geográficos, México.

Uribe Iniesta, Rodolfo (2003), La transición entre el desarrollismo y la globalización: ensamblando Tabasco, Cuernavaca, Morelos: UNAM/CRIM.

Uribe Iniesta, Rodolfo (comp.) (1988), Introducción: medio ambiente y comunidades indígenas del sureste, Villahermosa, Tabasco: CONAMEX/UNESCO/SECUR.

Thompson, Roberto G. (s.f.), Conflictos campesinos e intervención estatal en torno a la explotación petrolera en el sureste de México: el área de Reforma Chiapas-Tabasco 19701980 [manuscrito].
Tudela, Fernando (1989) [1992], La modernización forzada del trópico: el caso de Tabasco. Proyecto integrado del Golfo, México: El Colegio de México.

Vargas Pacheco, Ernesto (1994), "Síntesis de la historia prehispánica de los mayas chontales de Tabasco-Campeche", en América Indígena, Historia Prehispánica, vol. 1 y 2, México: UNAM, pp. 15-61. Velázquez Guzmán, Ma. Guadalupe (1981), Movimientos campesinos y explotación petrolera. El caso del Pacto Ribereño [manuscrito], México: Centro de Investigaciones para la Integración Social.

West, Robert C., Norbert P. Psuty y B.G. Thom (1985), Las tierras bajas de Tabasco, México: Gobierno del Estado de Tabasco.

Zurita Pedrero, Carlos Mario (1998), Historia y Monografía de Jalapa, México: H. Ayuntamiento de Jalapa, Tabasco. 1998-2000. 